Voix et Images

voixetimages

\title{
Charles Guérin : le récit et la thèse
}

\section{André Sénécal}

Volume 5, numéro 2, hiver 1980

Yves Thériault

URI : https://id.erudit.org/iderudit/200210ar

DOI : https://doi.org/10.7202/200210ar

Aller au sommaire du numéro

Éditeur(s)

Les Presses de l'Université du Québec

ISSN

0318-9201 (imprimé)

1705-933X (numérique)

Découvrir la revue

Citer cet article

Sénécal, A. (1980). Charles Guérin : le récit et la thèse. Voix et Images, 5(2),

333-340. https://doi.org/10.7202/200210ar d'utilisation que vous pouvez consulter en ligne.

https://apropos.erudit.org/fr/usagers/politique-dutilisation/ 


\section{Charles Guerin: le récit et la thèse}

Dans une note qui sert de postface au roman, Pierre Chauveau nous confie qu'en écrivant Charles Guérin « il a essayé de peindre sur le tissu d'une simple histoire de mours de son pays. " L'auteur ajoute qu'il a

aussi écrit son ouvrage avec la double préoccupation que doivent causer à tous ceux qui réfléchissent à l'avenir du pays, l'encombrement des carrières professionnelles où se jette notre jeunesse instruite, et le partage indéfini des terres dans les familles de nos cultivateurs. S'il peut contribuer à attirer l'attention de tous les véritables patriotes sur l'ceuvre de la colonisation, il croira, sous une forme légère, avoir fait quelque chose de sérieux (375).'

Au total, Chauveau n'accorde qu'une part assez faible au probleme du partage des terres et à l'ceuvre de colonisation. Dans un bref épilogue, l'auteur se contente d'ajouter une fin édifiante et patriotique à son roman. Charles Guérin, son frère l'abbé Pierre el son meilleur ami, le médecin Guilbault entrainent plusieurs habitants vers les terres de défrichement où ils fondent une nouvelle paroisse. Voilà tout un programme utopique à peine ébauché et que Gérin-Lajoie reprendra dans Jean Rivard. D'abord et avant tout, Charles Guérin est un roman de moeurs, le roman des espoirs et des désillusions d'une entrée dans la vie.

A seize ans, à peine sorti du collège classique, Charles Guérin devient subitement l'espoir de sa famille. A sa mort. le père de Charles a laissé une succession encombrée, ce qui a permis à Wagnaër, un étranger, de s'emparer du commerce du défunt et de la plus belle part du patrimoine des Guérin. II convoite le reste de l'héritage, surtout la rivière aux Ecrevisses. L'ultime ambition du capitaliste est d'exploiter ce cours d'eau qui sillonne la terre paternelle. En attendant que ses deux fils. Pierre et Charles, prennent la relève. Mme Guérin résiste à l'intrus malgré des offres alléchantes. Mais Pierre, désabusé, pleinement conscient qu'il ne peut réaliser ses réves de vie créatrice en Bas-Canada, prend le parti de s'exiler. La responsabilité de redorer le blason familial incombe donc a Charles qui, sans beaucoup d'enthousiasme, se rend à Québec pour y étudier le droit. 
Le jeune homme devient vite une des figures les plus romanesques du milieu estudiantin de la Vieille Capitale. Ballotlé par les dernières chimères de son imagination, il dissipe son temps en poursuivant des rêveries qui flattent son amour-propre. II s'attache de plus en plus à Henri Voisin qui encourage sa course écervelèe vers le désenchantement. Voisin, c'est l'ambition, l'être fourbe brûlé par le désir de parvenir à tout prix. Instruit de la cupidité de Wagnaër, il gagne l'estime de Guérin pour mieux le trahir plus tard.

Charles tombe amoureux de Marichette, la nièce de son patron, M. Dumont. Ame franche et sensible, l'héroïne est capable d'un sacrifice émouvant. Quoiqu'elle connaisse les faiblesses de caractère du prétendant. elle s'engage sans retour à l'épouser mais elle l'exempte d'une promesse réciproque. Charles oublie vite celle qui l'attend. Mme Guérin, aveuglèe par l'amour-propre, croit deceler l'amour naissant entre son fils et Clorinde, la fille de Wagnaër. Séduit par la beauté de Clorinde et trop faible pour contredire l'ambition de sa mere, Charles se plie aux caprices du destin. Mme Guérin émancipe son fils et elle lui confie les derniers fonds de la famille pour qu'il puisse exploiter la rivière aux Ecrevisses.

Les espoirs du nouvel homme d'affaires s'effondrent vite. De connivence avec Voisin, Wagnaër compromet Charles dans une complexe affaire de billets. Ruinée, la famille assiste à la vente de la terre paternelle qui passera à l'étranger. Puis, notre héros apprend que Wagnaër destine Clorinde à Henri Voisin. La fille du marchand refuse de lui désobeir en s'enfuyant avec Charles et, plutót que d'épouser un être vil, elle prend le voile sous le nom de Sceur Charles. La famille Guérin se réfugie à Québec où la mère meurt, victime du choléra. Par un cruel jeu du sort. Pierre, devenu prêtre, revient juste á temps pour enterrer sa mère.

Le roman se termine sur une note providentielle. $M$. Dumont meurt en laissant sa fortune à Charles et à Marichette. Fidèle à sa promesse, celle-ci pardonne au héros et devient sa femme. Après quelques années, Charles fonde une nouvelle paroisse dans les terres de défrichement. II y attire plusieurs habitants qui s'apprètaient à émigrer aux États-Unis.

Lire Charles Guérin, c'est aussi découvrir la plupart des problèmes qui agitèrent la jeunesse sortant des collèges classiques à la veille de l'insurrection de 1837. En mettant en scène Charles et Pierre Guérin, Jean Guilbault et Henri Voisin, Chauveau nous propose un drame psycho-sociologique qui analyse les rapports essentiels entre les individus d'une classe sociale, celle des hommes de profession, et leur milieu, le Bas-Canada surpeuplé, transformé radicalement et irrévocablement par l'ascendance sociale et économique de l'Anglo-Saxon. Chauveau prend pleinement conscience de ce nouvel ordre. II en dénonce l'effet corrosif sur les valeurs traditionnelles de la socièté québécoise ; il déplore l'arrivisme et la démoralisation qui en résultent.

Quant à la réussite mondaine el au conflit économique entre les deux races, l'ambition des Guérin se solde par un èchec. Wagnaër s'empare de la rivière aux Écrevisses et Charles n'épouse pas Clorinde qui lui aurait ouvert 
les portes de la nouvelle société capitaliste et anglaise. Et la leçon idéologique est la suivante: l'arrivisme et l'abandon des valeurs traditionnelles mènent le Canadien français à la faillite matérielle et morale. La démonstration est mélodramatique. Wagnaër, incarnation de tout un système de valeurs économiques et morales que l'auteur censure, s'empare de la terre paternelle. Avec l'aide d'Henri Voisin, démon de l'ambition, l'envahisseur broie les Guérin.

La philosophie autant que les actions de Wagnaër exemplifient d'ailleurs sa nature carnassière: "Il faut tout tourner à son profit, sans se géner pour personne..." (53). Comme Balzac, Chauveau prête au personnage-type une physionomie qui sied au profil moral:

M. Wagnaër était un homme trapu, surchargé d'embonpoint: son visage était rouge, marqué de petite vérole, el comme frotté d'huile; son nez plat, ses sourcils épais et roux, ses yeux petits et ciroteux, ses lèvres épaisses, sa bouche très grande et laissant voir deux superbes rangées de dents qui auraient fait honneur a un animal féroce. Avec cette formidable mâchoire, M. Wagnaër aurait pu exploiter toute la création (55).

Le portrait et les gestes sont outrés; ils relévent de la caricature.

Henri Voisin. personnifie la déchéance morale du traittre ethnique. Converti au pragmatisme, il n'hésite pas a exploiter les siens et a réduire l'amour à de vils marchandages. Il est le porte-parole des âmes défaitistes à l'heure la plus périlleuse pour le salut de la race. En bon justicier omnipotent, Chauveau lui réserve une fin décevante. Il ne trouvera jamais la femme qui le rendra riche car “les années qui s'écoulent n'ajoutent point de charme á sa physionomie qui de laide est devenue affreuse, ni à ses manières, qui de communes sont devenues détestables " (344). Les derniers mots au sujet de Wagnaër sont aussi exemplaires: "La bonne fortune l'a abandonné et, au rebours des années passées, moins il a mis d'honnêteté dans ses marchés, moins ils lui ont réussi. Le remords, le dépit, l'ennui l'ont remis sur la voie d'anciennes habitudes d'ivrognerie... (343).

La leçon idéologique préordonne donc la déchéance de Wagnaër et de Voisin tout comme elle nous laisse anticiper le rachat exceptionnel de Charles Guérin. Le héros, guéri de son mal de siècle, sauvé de la ruine matérielle par la fortune providentielle de $M$. Dumont, devenu riche sans avoir trempé son áme dans la fange du capitalisme, accède à la nouvelle élite laïque qui s'insurge contre le conquérant et ses valeurs matérialistes. Ce destin édifiant et artificiel voile l'échec retentissant de la nouvelle classe que Chauveau défend. C'est Jean Guilbault, véritable patriote, qui annonce cette nouvelle elite: "Il y a une nouvelle noblesse, la noblesse professionnelle, née du peuple, qui a succédé a la noblesse titrée" (83). Pierre Guérin nous trace l'ambition de ces champions du peuple: "Je voudrais être dans ma localité le chef du progrès. Je voudrais établir quelque manufacture nouvelle, arracher pour des pauvres gens un peu de l'argent que l'on exporte tous les ans en échange des produits démoralisateurs de l'étranger " (69). 
Or, malgré l'altruisme et le paternalisme qui dictent ce rêve populiste à Chauveau, celte nouvelle noblesse n'est pas du peuple; elle en est séparée par l'éducation et la conscience de classe. Charles cherche une évasion romantique à la campagne; il est impropre aux travaux des champs. Le terme que les habitants emploient pour le designer, " le Monsieur de la ville, " tout comme l'échappée révélatrice de Pierre Gagnon à Jean Rivard, ("Que vous êtes heureux, mon Empereur, de savoir lire...".), ${ }^{3}$ nous montre toute ta distance entre cette nouvelle élite lettrée et le peuple des campagnes.

La nouvelle classe appartient á la bourgeoisie mais le collège classique ne l'a pas préparée à en accepter les valeurs. Elle ne peut donc pas s'intégrer aux nouvelles structures économiques qui façonnent le continent. Le térme de Fernand Ouellet est juste : cette noblesse est composée de * déracinés. „Et c'est en empruntant l'optique de cet historien qu'il faut apprécier l'élaboration mythique du roman:

Les prolessions libérales étaient mal préparées à assumer leur rơle d'élite. Elles n'étaient pas suffisamment libérées des traditions paysannes pour admettre et apprécier l'importance des changements qui s'imposaient tant au niveau des valeurs que des institutions du Canada français. Au reste, leur formation classique et abstraite, trop appuyée sur le passé et pas suffisamment accueillante au réel, avait été un pauvre instrument de promotion sociale. Aussi, les hommes de profession, dans trop de cas, faisaient-ils figure de déracinés et d'individus mal adaptés au monde bourgeois auquel désormais ils appartenaient... . Méme s'ils se révèlent éminemment-sensibles aux déséquilibres du temps, ils ne parviennent pas à decouvrir une solution fructueuse aux problemes concrets qui s'imposent a leur attention.4

Ouellet conclut: «Aussi, la situation leur apparaît-elle sous forme de périls collectifs ... . Le premier de ces périls, c'est le capitaliste anglais, c'est même le capitalisme tout court... . 5

Chauveau, apologiste de sa classe sociale, illustre nettement cette position défensive et anachronique dans la mesure oủ ses pèrsonnages (ou le narrateur) associent tous les malaises de l'époque à la prépondérance de l'Anglo-Saxon. L'auteur prend pleinement conscience de l'encombrement au sein des professions libérales, mais loin d'attribuer la situation aux collèges classiques qui refusent de préparer l'élite au nouvel ordre, il s'en prend au gouvernement colonial:

Sous tout autre gouvernement que sous le nôtre, les carrières ne manquent pas à la jeunesse.

Ici, au contraire, c'est l'exception qui fait la règle. L'armée et sa gloire bruyante, si belle par là même qu'elie est si péniblement achetée; la grande industrie commerciale ou manufacturière, que l'opinion publique a èlevée partout au niveau des professions libérales $[\ldots]$; le gènie civil, les bureaux publics, la carriere administrative [...] voila autant de perspectives sédussantes qui attendent le jeune français au sortir de son collège. Pour le jeune Canadien doué des mèmes capacités, et à peu près du même caractère, rien de tout cela! (36) 
Même le caractère d'Henri Voisin est attribué à l'effet corrupteur de l'étranger plutôt qu'à une laille morale:

La corruption qui faisait de si rapides progrès dans l'âme de Henri Voisin, était donc le résultat de la mème maladie sociale qui avait chassé Pierre Guérin loin du toit paternel. Parmi les infortunés jeunes gens que le malheur de notre condition présente et les préjugés inhèrents à cette condition forcent chaque année à faire un choix entre l'état ecclésiastique et trois autres professions encombrées au-delà de toute mesure, quelques-uns, en effet, s'épouvantent, se désespèrent, s'enfuient; d'autres hésitent et tâtonnent longtemps pour n'arriver à rien; d'autres se consument honnêtement et laborieusement dans l'obscurité et la misère; d'autres enfin se jettent à corps perdu dans le charlatanisme et l'intrigue. L'émigration forcée, l'oisiveté forcée, la démoralisation forcée, voilà tout ce que l'on offre à notre brillante jeunesse... (109).

Désabusé, victime de sa pensée anachronique, Chauveau attribue le drame de son époque uniquement aux suites de la conquête. En réponse au péril collectif qu'il croit discerner, il se replie vers le passé et identifie la survie de la race a l'agriculturisme et au messianisme. 6

Le dénouement invraisemblable du roman est un manifeste idéaliste qui voile l'echec des héritiers de 1837, échec parfaitement illustré par l'intrigue jusqu'au dénouement miraculeux qui estompe la causalité d'un récit réaliste. Mais méme le dénouement artificiel trahit l'intention de l'auteur. Comme Jean-Charles Falardeau l'a bien démontré, le repli du héros vers les terres de colonisation masque le huis clos de la société québécoise de l'époque:

Pour coux qui acceptent d'y vivre, la socièté canadienne apparaît comme un cul-de-sac. Le héros de roman doit, pour réussir, reculer d'où il est parti pour se diriger ailleurs où tout est à reprendre depuis les débuts. [Charles Guérin] ...ne fait de sa vie une réussite qu'une fois qu'il a quitté son milieu québécois, une fois qu'il est rendu dans les terres neuves montréalaises.'

II n'y a pas que l'intrigue principale et les protagonistes du mal qui soient assujettis aux exigences d'une these. L'intrigue sentimentale et ses acteurs eux aussi illustrent la leçon du romancier. Le héros nous intéresse mais il nous est peu sympathique puisque, comme René, Charles Guérin incarne un mal du siecle que Chauveau baptise "maladie de l'intelligence." Gráce au don d'observation el à l'art de l'écrivain on peut découvrir dans le portrait de Charies la jeunesse idéaliste et désemparée qui sortit des collèges classiques entre 1830 et 1850 . Malgré son rachat exemplaire aux derniers chapitres, le héros demeure une âme romanesque, un être passionné qui cède à ses elans d'adolescent, emballé par de brusques mouvements d'enthousiasme mais vite désabusé par le défi du quotidien. C'est un petit mónsieur qui fait la cour à Marie Lebrun (Marichette). Charles Guérin domine ensuite un monde prosaique y promenant un dedain aristocratique qui fait partie de son hérilage: séduit par la beauté de Clorinde, il oublie vite Marichette qu'à première vue il avait considérèe "bien au-dessous de lui" 
(130). Chauveau a beau gloser sur l'irrésolution de son personnage, sur l'influence de sa mère, il ne parvient pas à atténuer l'étourderie et la faille morale de son héros. L'auteur qui a campé son protagoniste dans une pose romantique veut a la fois exploiter le pathos de son sort et réprouver sa " maladie de l'intelligence. " Le lecteur s'apitoie sur la victime des rapaces, sur le jouet des passions mais il ne peut oublier l'orgueil et l'insouciance qui font de Charles une áme fragile et superficielle.

C'est la thése idéologique qui dicte au héros son dernier rôle dans l'intrigue sentimentale et qui ruine la cohérence psychologique du personnage. On ne peut accepter qu'aprés avoir véritablement aimé Clorinde. Charles connaisse une dernière révélation et qu'en marge de son redressement moral, il reconnaisse la primauté de sa passion envers Marie Lebrun. L'équivoque enlève toute noblesse d'âme au héros. Chauveau sacrifie la vraisemblance romanesque aux impératifs de sa thèse. La contradiction ne se résout donc qu'au niveau de la lecture idéologique du récit. Charles Guérin épouse Marichette et non pas Clorinde Wagnaër parce que le couple doit illustrer les imperatifs de conservation de l'ethnie. Aux yeux du romancier, il était donc inévitable que le héros, personnification de la " nouvelle noblesse, " épouse une campagnarde et qu'ensemble ils établissent une petite patrie aux confins du territoire québécois. II est donc nécessaire que Chauveau purge son héros de sa maladie de l'intelligence, qu'une fortune sans souillures le sauve de la ruine matérielle, qu'une héroïne cornélienne le rachète d'une dèchéance morale.

En derniere analyse, les ressorts de l'intrigue sentimentale n'obéissent pas aux lois de la passion. II y a bien du romanesque dans l'éveil de l'amour de Marichette, dans l'idylle de Clorinde et de Charles mais finalement, ce romanesque fait place au déterminisme moral qui, lui, dépend de la démonstration idéologique. Selon la logique du sentiment, quoi de plus prédestiné que le couple Charles et Clorinde. Ce sont des âmes-sœurs qui vivent sous le signe de l'insouciance. Leur amour s'avive d'une méme sensibilité exacerbée par le rêve et l'abandon aux chimères de roman. La fatalité qui les sépare ne provient pas des caprices du sort ni même de l'irrémédiable du vceu de Clorinde à sa mère. Comme dans Attala, le lecteur savoure d'autant mieux les charmes du malheur que la fatalité est voulue par la victime, que Clorinde obeit à un serment qui ne l'engage pas selon la lettre du catholicisme. En prenant le voile qui la sauve du joug paternel, Clorinde obéit à un des imperatits qui préservent l'ethnie québécoise. Scur Charles, tentation romanesque de l'étrangère, née d'un père qui représente tout le système de valeurs matérialistes de l'Anglo-Saxon, ne peut rejoindre son amant qu'en illustrant héroïquement le systeme de valeurs spirituelles du Canadien français.

La constance de Marichette est aussi paradoxale que le geste fataliste de Clorinde. C'est d'abord le jeu des passions qui la lie a Charles; mais une fois que le sentiment aura engagé son âme, elle purifiera sa passion en restant fidèle à une promesse qui a la force d'un mariage éternel. II y a quelque chose de contradictoire dans le portrait de Marichette, une ame 
simple qui " ne connait pas les façons du grand monde" (128) mais qui, au couvent, "avait appris une foule de choses qui contrastaient singulièrement avec sa position" de fille d'habitant (125). Marichette qui raisonne pour se connaitre, qui, au sens cornélien du mot, est pleinement consciente de sa gloire, n'en accueille pas moins les premiers élans amoureux de Charles malgré "le beaucoup de poésie et de roman" de sa ferveur (154). Le personnage est assez romanesque pour succomber a l'amour fatal, mais trop héroique pour en connaitre les affres. Marichette incarne lamour dont l'essence est d'être irrévocable. Nous avons le vertige devant son suicide émotionnel. Sa grandeur d'âme vient de pardonner à un amant infidèle non pas parce qu'il le mérite mais parce qu'elle a déjà métamorphosé sa passion en noblesse d'áme.

Chauveau est divisé entre ses deux personnages principaux. Les ayant confrontés, il ne peut raccorder l'héroisme cornélien avec le mal du siècle. Le narrateur a beau nous confier qu'elle «lui pardonna beaucoup parce qu'il avait beaucoup aimé, et qu'il semblail disposer à aimer d'avantage" (339), le lecteur n'oublie jamais que Charles aime d'abord Clorinde Wagnaër. Le héros doit son rachat a deux femmes: Clorinde qui prend le voile pour lui rester fidèle et Marichette qui sacrifie son amour-propre.

Les personnages de Chauveau se laissent entrainer par les mouvements de leur sensibilité mais la passion, dans le contexte des valeurs traditionnelles de la société québécoise, n'est justifiable que si elle conduit au renoncement du moi en faveur des absolus de la race. Dictée par la thèse idéologique, la passion conduit donc à l'héroïsme et au sacrilice. Bien avant Maria Chapdelaine ou même Blanche d'Haberville, Chauveau répond à l'atavisme.

L'auteur de Charles Guérin est sans aucun doute le premier écrivain québécois qui possède les qualités essentielles du romancier de métier. Grâce à son don d'observation, il fait vivre des personnages qui intéressent. Son instinct dramatique peut transformer le réel en romanesque captivant; l'auteur sait tirer tout ce qu'une situation a de tragique ou de representatif. Avec un recul de plus de cent ans et l'alinéa de la Révolution Tranquille, on peut mesurer tout le parti pris et l'insuffisance de la thèse de Chauveau, thèse qui inéluctablement affaiblit la charpente réaliste de l'ceuvre. Mais là encore. malgré lui, le romancier reste le témoin fidèle de son époque puisque même dans ses fausses solutions et l'exemplarité de ses personnages, le roman trahit les aspirations et les dilemmes de l'élite du temps, l'insuffisance et la faillite de ses solutions. 
1. La pagination renvoie a l'édition la plus récente: Charles Guérin/Roman de moeurs canadiennes, édition présentée et annotée par Maurice Lemire (Montréal, Fides, 1978).

2. Pour de plus amples informations sur l'idéologie nationaliste de l'époque, voir: Fernand Ouellet, * Nationalisme canadien-français et taicisme au XIX siecle, * dans Jean-Paul Bernard, Les ldélogies québécoises au $19^{\circ}$ sidc/o (Montréal. Les Editions du Boréal Express. 1973), p. 37-60.

3. Antoine Guérin-Lajoie, Jean Rivard, le delrichour (récil de le vie réelle) suivi de Jean-Aivard economiste (Montréal, Hurtubise-HMH, 1977), p. 72.

4. Fernand Ouellet, op. cit. p. 48-49.

5. Ibid., p. 49.

6. C'est Michel Brunet qui a défini l'adaptation québécoise des doctrines physiocratiques en employant le terme, * agriculturisme. . Voir * Trois Dominantes de la pensée canadienne-française : l'agriculturisme, l'anti-étatisme el le messianisme : essai d'histoire intellectuelle, "Ecrits du Canada français. III (1957), p. 31-117.

7. "Le Désir du départ dans des romans canadiens du XIX siecle = Notre Société et son roman (Montreal, Hurtubise-HMH, 1967), p. 44. 